\section{Histoire Épistémologie Langage}

42-1 | 2020

La grammaire arabe étendue

\title{
Sur les traces de la racine trilitère dans la grammaire hébraïque
}

Judith Kogel

\section{(2) OpenEdition \\ Journals}

Édition électronique

URL : https://journals.openedition.org/hel/486

DOI : $10.4000 /$ hel. 486

ISSN : 1638-1580

Éditeur

Société d'histoire et d'épistémologie des sciences du langage

\section{Édition imprimée}

Date de publication : 28 septembre 2020

Pagination : 33-47

ISSN : 0750-8069

\section{Référence électronique}

Judith Kogel, « Sur les traces de la racine trilitère dans la grammaire hébraïque », Histoire

Épistémologie Langage [En ligne], 42-1 | 2020, mis en ligne le 28 octobre 2021, consulté le 29 octobre 2021. URL : http://journals.openedition.org/hel/486 ; DOI : https://doi.org/10.4000/hel.486

Ce document a été généré automatiquement le 29 octobre 2021.

\section{(c)}

$\mathrm{HEL}$ is licensed under a Creative Commons Attribution-NonCommercial-NoDerivatives 4.0 International License 


\title{
Sur les traces de la racine trilitère dans la grammaire hébraïque
}

\author{
Judith Kogel
}

\section{Introduction}

$1 \mathrm{Au}$ tournant $\mathrm{du} \mathrm{XI}^{\mathrm{e}}$ siècle, l'on assiste à une véritable révolution de la grammaire hébraïque. Abu Zakariyya Yahya ibn Dawud, connu sous le nom de Judah Ḥayyuj (Fez, 950 - Cordoue, $c a$ 1000), emprunte à la tradition arabe la notion de trilitéralité des racines. Pour la mettre en œuvre, il doit faire preuve d'une grande créativité, en raison $\mathrm{du}$ comportement des consonnes faibles. Il est, en effet, le premier à noter qu'elles peuvent ne pas être visibles dans certaines formes verbales, tout en restant présentes dans la forme théorique de base. Il reste, cependant, une difficulté majeure pour les apprenants : comment identifier la racine d'une forme nominale ou verbale complexe? Dans un chapitre original de sa grammaire, le $M a^{\prime} a s e h ~ e f o d^{1}$, Profiat Duran (Perpignan, $<1360$ - Catalogne, ca 1414) propose des techniques didactiques d'identification, systématiquement reprises dès la Renaissance par les humanistes chrétiens et ce, jusqu'au XIX ${ }^{e}$ siècle.

\section{Une révolution dans la grammaire hébraïque}

2 Vers la fin du $x^{e}$ siècle, Judah Ḥayyuj, qui vivait à Cordoue, rédige deux traités de morphologie en arabe (caractères hébreux) dans lesquels il propose un modèle théorique permettant de décrire et d'expliquer toutes les formes verbales irrégulières de l'hébreu biblique : le Kitāb al-af'al dِ awāt hurūûf al-līn (traité des verbes contenant des lettres faibles) et le Kitāb al-af'al d̂awāt al-mițlayn (traité des verbes contenant des lettres doubles) ${ }^{2}$. L'auteur innove sur deux points: il postule l'existence d'une racine consonantique servant de base dérivationnelle, qu'il est possible d'induire et de reconstruire à partir des mots attestés dans la Bible et il affirme que toutes les racines des verbes sont trilitères, "il n'y a pas de verbes contenant moins de 3 consonnes » 
(Hayyuj $1870: 12 ; 1844: 27$ ), même lorsqu'elles ne sont pas toutes apparentes ${ }^{3}$. C'est là que réside l'originalité de son analyse car si la trilitéralité de la racine est fortement influencée par la tradition grammaticale arabe ${ }^{4}$, Hayyuj a dû faire preuve d'une grande créativité pour l'adapter à la morphologie hébraïque. Rappelons qu'en arabe, les trois radicales sont presque toujours apparentes et relativement aisées à retrouver quand elles ne le sont pas, ce qui n'est pas le cas en hébreu.

Jusque-là, les écoles linguistiques partageaient une conception minimaliste de la racine. C'était, pour elles, un lexème/sémantème composé des consonnes qui restaient visibles dans les différentes formes verbales; on avait ainsi des racines à 1,2 ou 3 consonnes. Le traité grammatical, Mahberet, de Menahem ben Saruq (Tortose ca 910 - Cordoue ca 970) reflète cette conception de la racine ${ }^{5}$ tout en l'organisant selon l'ordre alphabétique.

Avant que la trilitéralité des racines ne soit établie, les exégètes qui éprouvaient quelques difficultés à identifier les racines de certains mots usaient de subterfuge pour en indiquer le sens. C'est notamment le cas du célèbre Salomon ben Isaac, dit Rashi (1040-1105), qui renvoyait fréquemment à la traduction araméenne accompagnant traditionnellement le texte biblique dans les manuscrits ashkénazes, quand il ne parvenait pas à discerner la racine d'une forme verbale et souhaitait néanmoins l'expliciter. Rashi commente ainsi le mot wa-yyåronnū (Lv 9,24), également analysé par Ḥayyuj, kə-targumo "comme le Targum d'Onqelos [l'a traduit]», qui renvoie à l'araméen wə-šabbåḥ̂, « ils louèrent ». De fait, le Targum traduit toutes les occurrences de la racine hébraïque RNN par des formes équivalentes de la racine ŠBḤ, une indication qui a assurément échappé à Rashi. S'il avait pu parcourir l'ensemble de la Bible, avec en vis-à-vis la version du Targum, il aurait été en mesure de rapprocher cette forme verbale d'un autre mot de la même racine.

C'est en examinant les formes verbales irrégulières que Ḥayyuj a réussi à établir que la forme phonologique (morpho-phonétique) de base peut être altérée lors de la dérivation. Ce phénomène avait induit en erreur ses prédécesseurs qui avaient considéré qu'il pouvait exister des racines biconsonantiques, voire même monoconsonantiques. Dans un second temps, le grammairien a décrit le comportement des consonnes faibles de l'hébreu et a noté que les lettres alef, he, waw et yod ont un statut particulier: elles peuvent ne pas être prononcées, se substituer les unes aux autres, être supprimées ou assimilées. La lettre devient ainsinנעלם une [lettre] quiescente cachée (sākin layyin)", qui peut disparaître graphiquement tout en faisant partie de la racine. Il convient, dès lors, d'être attentif aux variations vocaliques, notamment l'allongement de la voyelle précédente, qui peut constituer une trace de leur existence et à la présence de dageš compensatoire. Ḥayyuj a ensuite exposé quelques règles générales permettant l'identification des racines :

[...] et yissob (Gn 42,24) yåhom (Is 44,16) et autres formes semblables ressemblent à yåšūb $(\mathrm{Lv} 13,16)$ et yåqūm (Ex 21,19) et autres formes sœurs, et on peut les confondre [...] et en retrouvant pour chacun des deux types le sens primordial et la racine, on comprend la différence. De plus, les unes sont marquées d'un dageš et la lettre géminée est visible en ce qu'elle est absorbée par la seconde puisqu'on dit wayyåsobbū (Jos 6,14), wa-yyåronnū (Lv 9,24). Les autres sont marquées d'un rafeh [trait suscrit qui marque la prononciation fricative de certaines consonnes, en quelque sorte le contraire du dageš] puisqu'aucune lettre n'a été absorbée et les waw que [ces formes verbales] contiennent correspondent à la deuxième consonne de la racine, comme wa-yyåqūmūu (Gn 24,54), wa-yyåšub $\underline{u}$ (Jg 8,33). Ainsi, tu comprendras que si ces formes sont marquées d'un dageš ${ }^{6}$ lorsqu'on ajoute d'autres lettres (suffixes), il s'agit d'un verbe géminé comme wa-yyåsobbū, wa-yyåronnū $(\operatorname{Lv} 9,24)$ et 
wə-hinneh təsubbēnåh (Gn 37,7). Et s'il y a un rafeh, il s'agit d'un verbe dont la deuxième radicale est faible, comme wa-yyåqūmū (Gn 24,54), wa-yyåšūbu $\bar{u}(\mathrm{Jg} 8,33)$ et tåšobnnåh (I S 7,14)

6 C'est à ces méthodes permettant d'identifier les racines comportant des consonnes faibles que nous allons nous intéresser, et à leur devenir dans les ouvrages postérieurs. Peu de grammaires hébraïques en contiennent alors qu'elles figurent dans un nombre non négligeable de grammaires latines de l'hébreu.

\section{Les grammaires hébraïques médiévales}

7 Abraham ibn Ezra (ca 1089 - ca 1167), l'un des traducteurs des traités grammaticaux de Ḥayyuj, a introduit quelques règles générales (kəlal) dans son premier ouvrage linguistique, Sefer șahot ${ }^{8}$, dont voici une des entames : «Et je vais maintenant te donner une règle qui te permettra d'analyser les formes verbales et d'en identifier la racine » (Lippmann 1827 : f. 48r). Aussi étonnant que cela puisse paraître, on ne retrouve pas de démarche de ce type dans les ouvrages linguistiques les plus célèbres, qu'il s'agisse de ceux de Jonah ibn Janah, qui a composé les premiers traités ${ }^{9}$ comportant toutes les bases théoriques de la grammaire hébraïque et qui est considéré comme le continuateur de Ḥayyuj, ou de ceux de la famille Qimhị, Joseph, Moïse ou David ${ }^{10}$.

8 En revanche, Profiat Duran a consacré un chapitre entier du Ma'aseh efod aux techniques à employer pour isoler ou retrouver les trois lettres radicales. Médecin, astrologue, mathématicien et philosophe, Profiat Duran appartenait à l'élite socioculturelle de la communauté juive dont on a de nombreux exemples ${ }^{11}$, et comme ses pairs, il fut aussi un enseignant reconnu. Même après sa conversion forcée au christianisme, en 1391, Profiat Duran, appelé dès lors Honoratus de Bonafide, continua d'enseigner ${ }^{12}$ et de composer des ouvrages ${ }^{13}$. C'est avec le $\mathrm{Ma}^{\text {'aseh }}$ efod, que l'on pourrait peut-être traduire "[Grand-] œuvre du efod ${ }^{14}$ ", composé en 1403, que la carrière littéraire de Profiat Duran s'achève.

9 Ce passage, intitulé bi-netinat derakhim yud'u mehem šoršei ha-pe'alim we-ha-šemot u-milot ha-ta'am ha-nimșa'im ba-katub, «À propos des moyens permettant d'identifier les racines des verbes, des noms et des particules présents dans la Bible» (Friedländer 1865: 141-145), figure après la grammaire du nom et du verbe. Il s'ouvre sur l'importance et la nécessité qu'il y a à identifier les racines des mots bibliques, et se poursuit avec un véritable cours de méthodologie en huit points qui détaillent les différentes techniques à mettre en œuvre :

1. [...] Seules «les lettres qui sont toujours présentes dans le nom et les formes dérivées, soit en acte comme c'est le cas pour les verbes réguliers, soit en puissance comme c'est le cas pour tous les autres types de verbes, sont appelées « radicales » de cette forme et de tout ce qui en dérive ». Il rappelle ensuite que la forme de l'accompli masculin, troisième personne du singulier, au $q a l^{15}$ et au pi ${ }^{\prime} e l$ ne contient que les lettres de la racine, «et c'est une des manières qui permet d'identifier les racines".

2. Pour identifier les racines, il convient « d'examiner toutes les formes existantes [dans le texte biblique] pour tenter de trouver une preuve de la racine ».

3. «Il faut être attentif à la présence ou l'absence de dageš, s'il ne s'agit pas d'un verbe régulier qu'il est facile d'appréhender, comme nous le verrons. Si l'on repère un dageš, même s'il n'est pas présent à toutes les formes, on doit en déduire que l'on 
a affaire à une racine géminée ». Il signale ensuite que Ḥayyuj avait déjà indiqué ce moyen d'identifier la racine dans le chapitre consacré aux géminés ${ }^{16}$.

4. «Le quatrième moyen est de s'aider de la vocalisation pour une partie des cas, puisqu'à l'accompli, seule la voyelle notée sous la lettre initiale de la racine permet, au pa'al, de faire la différence entre les verbes 'ayin-waw [verbes dont la deuxième radicale est un waw] et les géminés: un qameș pour compenser la lettre faible disparue [verbes 'ayin waw] et un patah pour les verbes géminés ».

5. « Et la cinquième façon d'identifier les racines, c'est de comprendre la structure [du verbe] et c'est le principe essentiel pour en déduire les racines [...] Et c'est la raison pour laquelle les opinions des premiers grammairiens ont divergé concernant certaines racines et chacun a suivi son opinion concernant la structure [du verbe] et certains n'ont peut-être pas accédé à la vérité parce que la structure leur a échappé ».

6. «Et la sixième façon concerne les racines des noms dont on ne sait comment ils fonctionnent et varient dans le texte biblique mais s'il se trouve un verbe dénominatif et selon ce que l'on trouvera concernant le verbe, on en établira la racine du nom [...] De même pour dåg 'poisson', étant donné que l'on a wə-yidggū lårob , 'qu'ils foisonnent abondamment' (Gn $48: 16$ ), nous comprenons qu'il a une troisième consonne faible et non une deuxième comme l'ont cru certains [...] Et certains noms nous renseignent sur d'autres [...] et nous avons statué, [concernant la racine], pour ḥåron par analogie avec gåron ${ }^{17}$ ».

7. « Et la septième façon, [c'est d'] examiner les noms et les verbes avec [des formes] bibliques semblables qui te semblent plus évidentes et cela te permettra d'identifier la racine cachée mais il faut se garder de toute erreur : il peut souvent arriver que tu voies deux mots dont le schème se ressemble et que tu veuilles substituer les racines [de façon erronée] ».

8. « Et la huitième façon consiste à examiner le contexte où apparaissent les noms et les verbes d'une même racine. Et avec discernement et entendement, tu détermineras le sens de ce verset et selon ce qu'il t'apparaîtra de l'interprétation du verset, tu statueras à propos de la racine mais c'est une méthode à posteriori car la connaissance de la racine précède la compréhension du verset [...]».

Ce qui frappe dans ce texte, ce sont les éléments qui permettent une étude individuelle autonome, en l'absence de maître. Il s'agit d'une démarche inhabituelle et novatrice qui sera largement reprise par Sancte Pagnini dans son ouvrage Hebraicarum Institutionum libri IIII' ${ }^{18}$.

\section{La grammaire de Sancte Pagnini}

11 En 1526, paraît à Lyon, aux presses d'Antoine du Ry, la première grammaire hébraïque en latin, d'importance, publiée en France ${ }^{19}$. L'auteur, Sancte Pagnini (Lucques, 1470 Lyon, 1541), a été l'élève, à Florence, d'un juif espagnol converti, Clément Abraham. Il dédicace cette grammaire à Federigo Fregosio, archevêque de Salerne, " pour pallier le fait que ce dernier n'avait pas de précepteur pour l'hébreu (quod idoneo carebas praeceptore ${ }^{20}$ ) ». Bien que Robert Estienne, éditeur de la seconde édition de la grammaire de Sancte Pagnini, présente cet ouvrage comme une traduction fidèle - fere transcripti du Mikhlol de David Qimhi ${ }^{21}$, certains passages ont été empruntés au Ma'aseh efod ${ }^{22}$ de Profiat Duran. Sophie Kessler-Mesguich a été la première à le signaler et à noter que 
Pagnini ne le nommait que rarement ${ }^{23}$ parmi ses sources, alors qu'il expliquait fréquemment le vocabulaire technique de la grammaire hébraïque, en reprenant les définitions ${ }^{24} \mathrm{du} M \mathrm{M}^{\text {'aseh }}$ efod. Dans les chapitres 4 et 5 du livre IV (Pagnini 1526) ${ }^{25}$, De modo inveniendi radices, hoc est themata, et primitiva omnium Hebraicarum dictionum et particulatim nominum, "À propos des moyens permettant d'identifier les racines complexes ${ }^{26}$ et primitives de tous les mots hébreux et en particulier des noms » (ibid. : 395-398) et De modo inveniendi radices verborum, «À propos des moyens permettant d'identifier les racines des verbes » (ibid. : 398-400), Pagnini a perfectionné la méthode du grammairien Profiat Duran en distinguant les noms des verbes et en réorganisant les huit points énoncés par son prédécesseur.

De fait, Pagnini insiste, comme son modèle, sur l'importance qu'il faut attacher à l'identification des racines: «La raison pour laquelle il faut trouver les racines des noms [...] parce que leur connaissance conduit dans une grande mesure à la compréhension de l'écriture, j'estime très utile et déclare de quelle façon on peut identifier les racines des noms et des verbes». Il poursuit en rappelant "qu'il faut garder en mémoire que les noms et les verbes peuvent être parfaits, quiescents, défectifs ou géminés » et que « le šoreš, à savoir la racine des mots, sont les lettres qui sont toujours présentes, en acte dans la prononciation comme [dans les mots] parfaits, ou en puissance à l'aide de voyelles royales ${ }^{27}$ ou d'un dageš pour les [verbes] quiescents, défectifs ou géminés ${ }^{28} »$.

Il rappelle ensuite la trilitéralité des racines et la constitution des formes verbales et des noms à l'aide des lettres serviles, avec de nombreux exemples :

Et nous avons souvent rappelé que presque tous les noms et les racines des verbes contiennent trois lettres. Et dans les noms parfaits simples, il n'y a pas de doute ni de difficulté, qu'ils contiennent seulement trois lettres radicales, comme

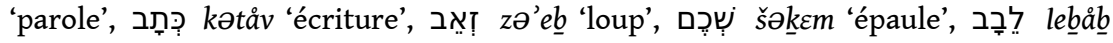
'cœur', Dans les autres cas, en revanche, qui comportent des lettres accidentelles et

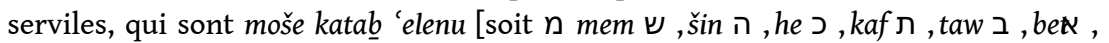
alef ל, lamed ',yod J, nun et I waw ] ${ }^{29}$, comme il a été dit au livre 1, chap.15, ou he'emanti [soit הe he alef $\mathrm{n}$, mem ], nun ת,taw et ' yod] ${ }^{30}$ pour les noms, une fois ces lettres enlevées, les trois lettres qui restent dans les noms parfaits sont les radicales ou bien ces lettres accidentelles se trouvent au début ou seulement à la fin ou au

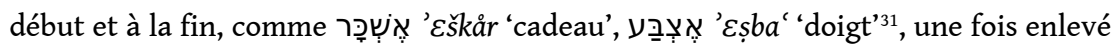

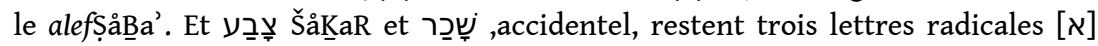

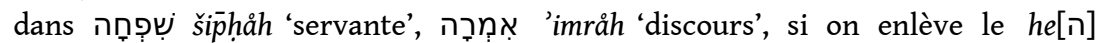
accidentel qui est la désinence du féminin, il reste les lettres radicales et אָָ 'åMaR.

Et dans lettre préformante mem et la lettre accidentelle [מ] [ה], [ [ [ ŠåPaḤ et eñ LåḤaM. Et de même, dans les autres [noms] composés avec les lettres accidentelles, il reste trois lettres radicales en acte.

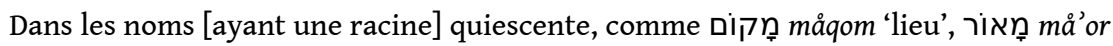
'luminaire', une fois enlevé le mem אוא accidentel, il reste les lettres radicales [מ]

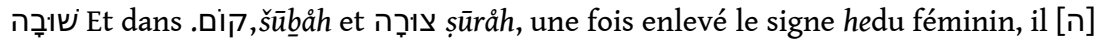

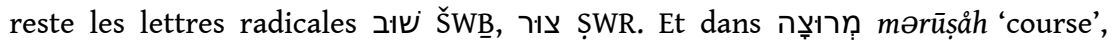

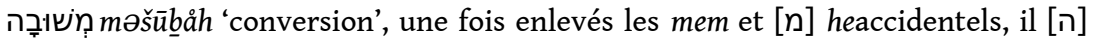
reste les lettres radicales רוּ RWS quiescents, les trois lettres radicales ne sont pas visibles en acte, comme dans l'exemple précédent, mais en puissance des "voyelles royales», qui ont en 
puissance les lettres quiescentes, comme sont les voyelles camés, séri, chiric, suivies d'un yod, cholem. Comme jịT zådon 'la superbe', une fois enlevées les lettres accidentelles vau et [1] nun Tị il reste, [] zåd et en puissance le camés est vauEt .[1] ainsi זו ZWD est la racine. Et dans Iִ zedon 'superbe', une fois enlevés pareillement vau et [1] nun Ț il reste, [] zed et le séri est vauen puissance et ainsi [1] la racine est זٓ ZWWD ${ }^{32}[. . .]^{33} \mathrm{Et}$ dans hănåpåh 'tamisage', une fois enlevés les deux he נָ accidentels, il reste [ה] nåp. Et le camés est le vauen puissance et ainsi [I]

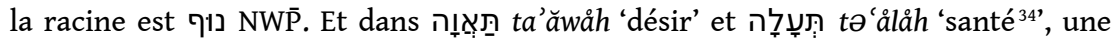
fois enlevé le tau åLåH. Et עָ עָלָ 'åWåH et occidentel, il reste les radicaux [ת]

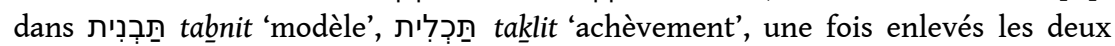
lettres tau et [ת] iod de la voyelle royale dans laquelle [1] heest en puissance, il [ה]

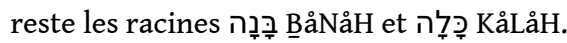

Dans les noms [ayant une racine] défective où il manque généralement le nunou [3] le iod que le ['] daghés ou une voyelle royale supplée, comme dans מַש massor 'scie',

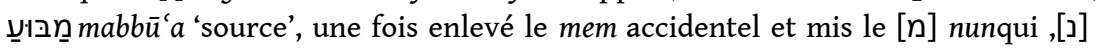

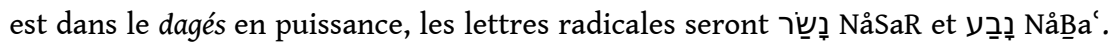

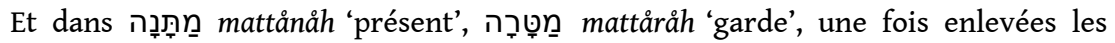

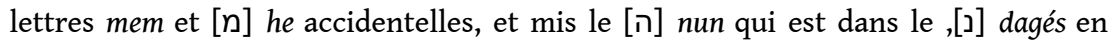

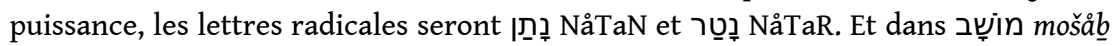
'enceinte', מוֹרָ moråš 'possession', une fois enlevées les lettres mem et [מ] vau[1] [accidentelles], et mis le iod qui est en puissance dans le ['] cholemles lettres, [i]

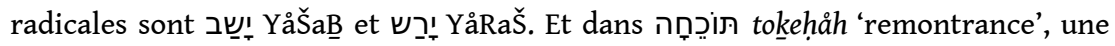
fois enlevées les lettres accidentelles tau et [ת] he et mis le, [ה] iodqui est dans le, ['] cholem שֵֶׁ Yå Yậ. Et dans puissance, les lettres radicales sont [i] šenåh 'sommeil', ָָ de de̊h 'science', une fois mis le iod qui est en puissance dans le ['] séri

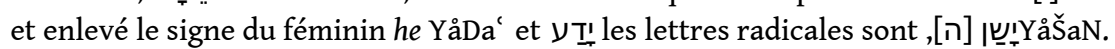

Dans les géminés parfaits, en revanche, c'est-à-dire dans ceux qui ont trois lettres radicales, il n'y a pas de possibilité de se tromper. Dans les autres verbes, il faut considérer que le daghés, qui supplée, fait fonction de vicaire des lettres défectives,

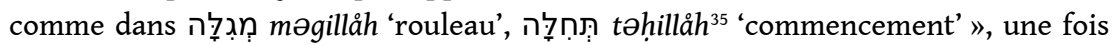
enlevées les lettres mem ou [מ] tau et [ת] hequi sont des lettres accidentelles, et, [ה] mis le lamed qui est en puissance dans le [ל] daghés, il reste les radicales et signe he du féminin et mis le [ה] tau et le [ת] memqui sont en puissance dans le [מ]

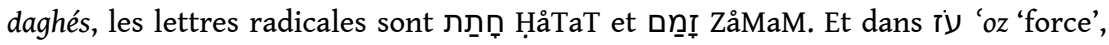
口n hom 'chaleur', une fois mis le [deuxième] zain et le [deuxième] [r] memqui [מ] sont en puissance dans le cholem, les radicales sont עָ 'åZaZ et

Ce long développement très didactique, qui ne figurait pas dans la grammaire de Profiat Duran, ne peut être dissocié des deux listes de noms qu'a insérées Pagnini en prologue au Thesaurus ${ }^{36}$ qui est, on le sait, une adaptation du Sefer ha-shorashim ou Dictionnaire des racines de David Qimhi : une première (3r-4v) où figurent les substantifs ordonnés en fonction des préformantes האמנתי - he, alef, mem, nun, taw, yod - et une seconde $(5 \mathrm{r}-5 \mathrm{v})$, rangée selon l'ordre alphabétique, faisant apparaître les racines des substantifs "défectifs [noms auxquels manquent une consonne de la racine sans compensation], quiescents [noms comportant une consonne quiescente cachée] et géminés ». Seul, le dernier paragraphe de ce chapitre 4 reprend fidèlement le point 6 du Efod, que nous avons résumé plus haut ${ }^{37}$.

De plus, s'il y a [dans la Bible] un verbe dénominatif, tu pourras juger du nom à partir de ce verbe. Nous disons que עָ ‘åš 'vers' est géminé, car nous trouvons dans

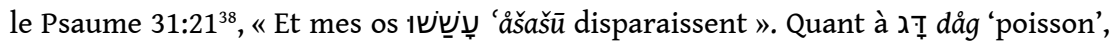
nous disons qu'il dérive de Tָָָ DåGåH non seulement parce que dans le pluriel, il n'y a pas de daghés [ce qui aurait été l'indice d'une racine géminée], mais aussi parce 


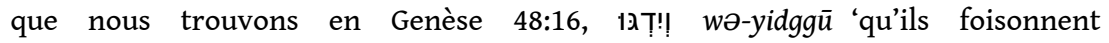
abondamment'. Et חָ̣ håron 'la colère', nous disons que le mot dérive du verbe

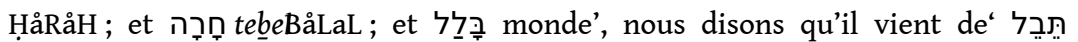

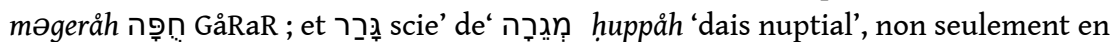
raison du daghés mais aussi parce qu'on trouve [dans la Bible] considérons qu'il fait partie des géminés. Les dissyllabiques avec daghés prouvent que les monosyllabiques dérivent des géminés, תפ pat 'morceau' et ק qal 'léger'

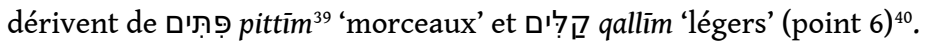

Le chapitre 5 est entièrement consacré aux verbes. L'auteur commence par traiter des verbes parfaits ou réguliers, verborum perfectorum, ceux qui gardent les trois consonnes de la racine à toutes les formes :

Découvrir les racines de verbes parfaits, qui ont toujours trois lettres radicales ne pose pas de difficulté quel que soit le binián. Une fois enlevées les lettres accidentelles, comme celles qui constituent les personnes, par exemple, ou les genres, ou les nombres, ou les modes, ou bien celles qui sont formatives des conjugaisons, il reste les radicales, qu'il est facile de voir dans les cas singuliers des binianím, c'est-à-dire les conjugaisons, mentionnés plus haut. Cependant, plus précisément, dans les verbes parfaits de première et seconde conjugaison, dans la troisième personne du masculin accompli, qui n'a aucune lettre accidentelle, se trouve la racine de toutes les formes qui dérivent d'elle, dont les personnes, le genre, le nombre et le mode. Et ainsi dans les verbes dont la première lettre est le iod quiescent: ou la dernière qui est, [י] alef ou [ [ [ heune fois trouvé l'accompli , ה] dont on a parlé, ou même l'impératif, outre le radical, ils n'ont pas de lettres

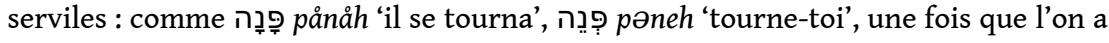

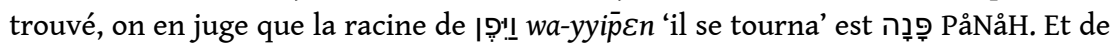

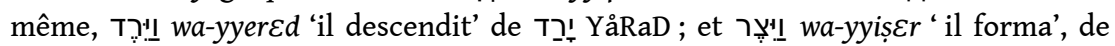

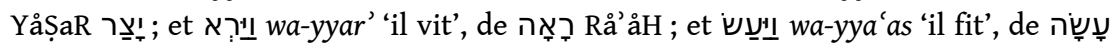
'åSåH, et de même pour les autres (point 1).

Mais dans les verbes dont la deuxième [lettre] est un vauquiescent, nous en ,[1] jugerons à partir du maqor ou du futur: dans ceux qui ont un I quiescent, à l'accompli ou au participe, dans ceux-là, le vauest seulement en puissance dans [1] la voyelle royale camés. Comme 

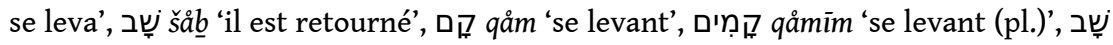

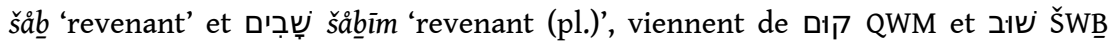
(point 2).

Dans les géminés en revanche, si on trouve un [verbe] complet, c'est-à-dire ayant trois lettres radicales, nous en déduisons les [racines des] imparfaits et défectifs.

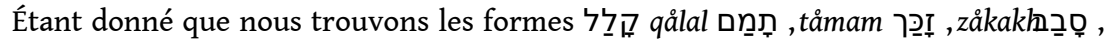

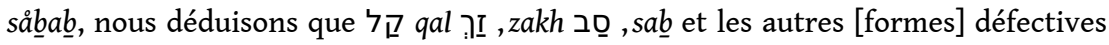
des verbes dont nous avons parlé, viennent de géminés - et ainsi pour les autres cas, dont nous avons parlé dans le livre 3, chap. 34 -bien que l'on puisse savoir qu'il s'agit de géminés grâce à la voyelle pathach, qui est propre aux géminés - comme le camés est propre aux verbes dont la seconde radicale vauest quiescente - et [1] grâce au daghés qui est dans la seconde radicale, comme dans 1 叉 qallū 'ils sont

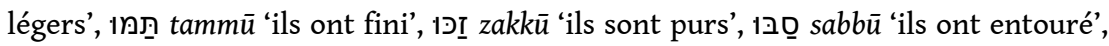
qui ont effectivement un daghés et sont des géminés. Même en ce qui concerne le futur des géminés, au binián niphal défectif, on sait grâce au pathach qu'il s'agit de futur des [verbes] défectifs quiescents, comme nous l'avons indiqué à sa place (points 3 et 4 ).

Il y a une autre façon d'identifier les verbes et les noms communs, nous devons faire l'effort de ne pas ignorer la signification des verbes et des noms. En effet (par 
exemple), que Tָ nåzad est 'il cuisinait', nous pouvons immédiatement déduire que

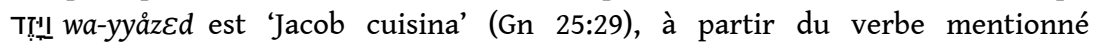
précédemment. De même, si l'on sait que אị 'ozen signifie 'oreilles' et le verbe dénominatif, 'percevoir avec les oreilles', ou 'incliner les oreilles', nous comprenons le verset Pv 17:4, «le menteur מֵזִין mezin (bien que le alef soit manquant) incline l'oreille vers la langue pernicieuse (dangereuse)» : on le déduit de אị 'ozEn et la

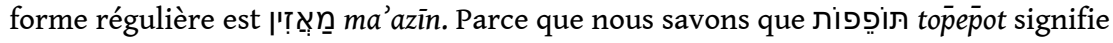

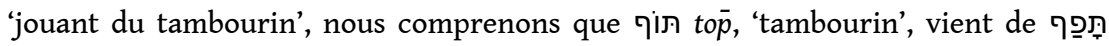
tåpa $\bar{p}$. Et nous pourrons de même discerner facilement les autres cas (point 5).

Il y a aussi une autre manière de reconnaître les [noms] communs, c'est-à-dire que par analogie, nous pouvons déduire ce qui n'est pas connu à partir de ce qui lui

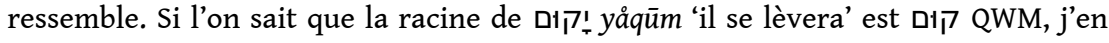

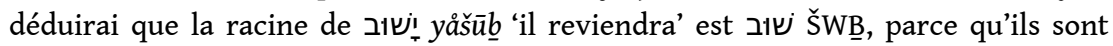
semblables et l'on doit étendre cette déduction aux autres cas similaires. Et de même pour les autres verbes et noms, comme אָָ åqūm 'je me lèverai' et åšū 'je reviendrai', et et

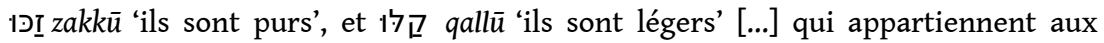
géminés. Mais cette méthode peut induire en erreur les débutants/ignorants, parce

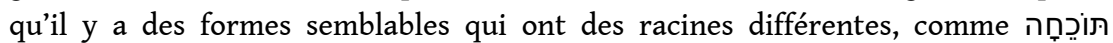
tokehåh 'remontrance', et

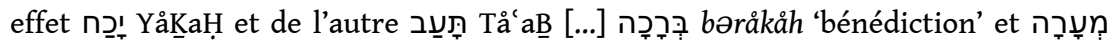

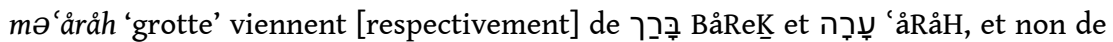
Må`aR, comme certains le pensent (point 5). C'est pour cette raison que ce mode de déduction demande une attention non moindre, pour que nous ne nous trompions pas dans les analogies. Mais nous venons de traiter quelques règles très utiles pour la compréhension des écritures sacrées (point 7).

$16 \mathrm{Au} \mathrm{XVII}^{\mathrm{e}}$ siècle, un autre hébraïsant chrétien, Johannes Buxtorf, résume en cinq points l'essentiel de cette démonstration dans l'introduction à son livre Epitome grammaticae hebraeae $e^{41}$, sous le titre Regulae de themate facile investigando et cognoscendo « Règles pour reconnaître et identifier facilement la racine » :

1. Si après avoir supprimé les lettres serviles, il reste trois lettres, celles-ci

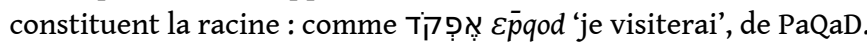

2. S'il n'en reste que deux, et que la première est marquée d'un dageš, alors ce dageš correspond le plus souvent à la première lettre radicale $]$ nun et plus rarement à '

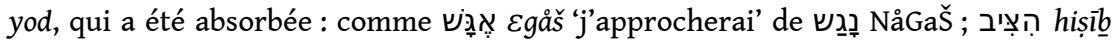
'il installa' de ְִ YåșaB.

3. Si c'est la dernière des deux qui restent, qui est marquée d'un dageš, il s'agit d'une

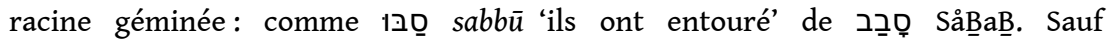
premièrement, si le dageš est un des traits de la conjugaison et que l'on peut identifier par la forme, dans ce cas il faut placer à la fin un he : comme ị gilū de GåLåH ; deuxièmement, si les noms avec suffixes pronominaux sont marqués d'un dageš dans la seconde lettre, ils ont pour consonne intermédiaire [deuxième

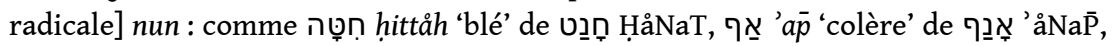
תבַּ bat 'fille' de BånåH.

4. Si la dernière des deux consonnes qui restent n'est pas marquée d'un dageš, soit la première lettre est un ' yod ou un $J$ nun, plus rarement un $N$ alef; soit la lettre intermédiaire est un I waw, soit la dernière lettre est un he et plus rarement un alef.

5. Si après avoir supprimé les lettres serviles, il ne reste qu'une lettre radicale, dans 
ce cas, il faut placer comme première lettre soit J nun, soit ' yod et mettre à la fin un הhe, excepté pour תֵ tet 'donner', dont la racine est נָָ NåTaN.

Il termine par ces mots: Quiconque deviendra un très bon grammairien n'aura pas besoin de ces règles ou d'autres semblables.

Ces règles sont versifiées dans l'édition Leusden ${ }^{42}$ (1691) et déplacées au chapitre 6.

1. Abjice Serviles, et tres si fortè supersunt,

Radicem thematis noveris esse tui.

2. Sin tantùm remanere duas conspexeris, adde

Nun vel Yod capiti, vel dato Wau medio,

Aut He postponas, aut conduplicato secundam

Vera statim Radix pullulat inde tibi.

3. Unica sed remanet mihi litera, quando removi

Serviles : Radix dic agè qualis erit?

Nun da principio, vel Yod superadde quiescens,

He fini : Radix inde petita venit.

4. Qui quid analogico normae dissentit ab usu;

Hoc exercitium, lector amice, dabit.

On les retrouve ensuite dans de nombreux ouvrages postérieurs. Adrianus Reeland (1721, p. [2]) les reprend en les faisant précéder du titre Investigationi Radicum plurimum inserviunt sequentes Versiculi. Dans une grammaire de l'hébreu, en anglais cette fois, James Noble (1832, p. 122) signale qu'il est « habituel de donner dans les grammaires hébraïques, ce que l'on qualifie de Règles pour identifier la racine ». Et, ajoute-t-il, « le fait est que la grammaire [hébraïque] n'est rien d'autre qu'un système de règles permettant d'identifier la racine. Ainsi, trouver la racine pour quelqu'un qui maitrise la grammaire n'est guère difficile; quant à celui qui ne la maîtrise pas bien, aucune règle sur le sujet ne sera d'une grande utilité ».

\section{Conclusion}

Ainsi, si les prémisses de ces passages didactiques permettant d'identifier les racines faibles sont déjà présentes dans les traités de Judah Ḥayyuj et dans le Sefer șaḥot d'Abraham ibn Ezra, elles ne sont devenues systématiques qu'au moment où l'enseignement de la grammaire hébraïque a franchi les frontières des communautés juives et ce, à mon sens, pour deux raisons : le processus d'apprentissage de l'hébreu débutait dès l'enfance sous la direction d'un maître qui guidait ses élèves et pouvait, en fonction de ses aptitudes pédagogiques, leur fournir les clés permettant d'identifier les racines; la connaissance intime des prières et des textes classiques facilitait le rapprochement entre mots dérivés de la même racine, dont l'un pouvait avoir conservé les trois radicales. Profiat Duran écrivit son ouvrage grammatical après avoir été converti de force en 1391, peut-être à destination de ses frères d'infortune qui ne pouvaient bénéficier d'un enseignement organisé, et il fut, semble-t-il, le premier à consacrer un chapitre entier à ce point. Sa démarche novatrice fut reprise par Sancte Pagnini dont les ouvrages ont influencé d'autres hébraïsants chrétiens, et ce jusqu'au $\mathrm{XIX}^{\mathrm{e}}$ siècle $^{43}$. Pour terminer, je souhaiterais mentionner un manuel français, celui de l'abbé Ladvocat, dont le titre confirme mon sentiment, à savoir que les règles énoncées par Profiat Duran avaient pour but d'aider un apprenant solitaire : Grammaire hébraïque à l'usage des écoles de Sorbonne, avec laquelle on peut apprendre les principes de l'hébreu, sans le secours d'aucun maitre ${ }^{44}$. 


\section{BIBLIOGRAPHIE}

\section{Sources primaires}

Benavente Robles, S. et Sáenz-Badillos, Á. Těšubot de los discípulos de Měnahem contra Dunaš ben Labrat. Grenade : Universidad de Granada - Universidad pontificia de Salamanca. 1986.

Buxtorf, J. Epitome grammaticae Hebraeae. Bâle : Ludovic Regis. 1629.

Buxtorf, J. et Leusden, R. Epitome grammaticae Hebraeae. Brittenburg : Luchtmans. 1691.

Díaz Esteban, F. Sefer 'oklah wě-'oklah. Colección de listas de palabras destinadas a conservar la integridad del texto hebreo de la Biblia entre los judios de la edad media. Madrid: Consejo superior de investigaciones científicas. 1975.

Friedländer, J. et Kohn, J. Maase Efod. Einleitung in das Studium und Grammatik der Hebräischen Sprache von Profiat Duran. Vienne : J. Holzwarth. 1865.

Ḥayyuj, J. ben David. Two treatises on verbs containing feeble and double letters. Trad. M. Gikatilla, éd. J. W. Nutt. Londres/Berlin : Asher. 1870.

Ḥayyuj, J. ben David. Grammatische Werke des R. Jehuda Chajjug aus Fetz. Trad. A. ibn Ezra, éd. L. Dukes. Stuttgart : A. Krabbe. 1844.

Ibn Janaḥ 1896 = Ibn Gānâh, A. M. Sepher Haschoraschim. Trad. J. ibn Tibbon, éd. W. Bacher. Berlin : H. Itzkowski. 1896.

Ibn Janah, J. Sefer ha-Riqmah (Kitāb al-Luma'). Trad. J. ibn Tibbon, éd. M. Wilensky, D. Téné, Ze'ev Ben-Hayyim. Jérusalem : Ha-Aqademyah la-lashon ha-'Ivrit. 1964.

Ladvocat, J.-B. Grammaire hébraïque à l'usage des écoles de Sorbonne; avec laquelle on peut apprendre les principes de l'hébreu, sans le secours d'aucun maître. Paris : Impr. de Vincent. 1755.

Lippmann, G. H. et Ibn Ezra, A. Sefer șaḥot. Fürth : Bi-defus D. Tsirndorfer. 1827.

Münster, S. Dictionarium Hebraicum. Bâle : Johann Froben, 1523.

Noble, J. Rudiments of the Hebrew language, with and without points. Glasgow : M. Lochhead. 1832.

Pagnini, Sancte. Habes hoc in libro... Hebraicas institutiones... quas nuper... Lyon : Antoine du Ry. 1526.

Pagnini, Sancte. Thesaurus linguae sanctae sive lexicon hebraicum. Lyon : Antoine du Ry. 1529.

Pagnini, Sancte. Hebraicarum institutionum Libri IIII. Paris : Robert Estienne. 1549.

Qimhii, D. Sefer Mikhlol. Éd. I. Rittenberg. Lyck : Petzall. 622 [1862] (réimpr. Jérusalem 1966). Qimhi, J. Sepher Sikkaron : Grammatik der hebräischen Sprache. Éd. W. Bacher. Berlin : M'kize Nirdamim. 1888.

Qimhi, M. Rabi Mose Kimhi in introductorio grammaticae. Haguenau : Thomas Anshelme. 1519. Qimhi, M. Sefer darkhe leshon ha-qodesh... : Liber V[iarum Lingu]e Sancte Rabi Mosse Kimahi. Éd. A.Giustiniani. Paris : Les Frères de Gourmont. [1520].

Reeland, A., Brevis introductio ad grammaticam hebraicam et chaldaicam. Éd. Charles Morthland. Glasgow : J. Duncan. 1721. 
Tissard, Fr. [Grammatica hebraica]. Paris : Gilles de Gourmont. 1508 ou 1509.

Sáenz-Badillos, Á. éd. Mahberet - Menahem ben Saruq. Grenade : Universidad de Granada. 1986.

Zsigmondy, S. Grammatica Hebraea. Vienne : Antonius Nob. de Schmid. 1828.

\section{Sources secondaires}

Bacher, W. 1882. Die grammatische Terminologie des Jehûdâ b. Dâwîd (Abu Zakarjâ Jahja ibn Dâud) Hajjû̀̄. Nach dem Arabischen originale seiner Schriften und mit Berücksichtigung seiner Hebräischen Übersetzer und seiner Vorgänger. Vienne : Gerold.

Emery, R. W. 1968. New light on Profayt Duran “The Efodi”. The Jewish quarterly review, $\mathrm{n}^{\circ} 58$ : 328-337.

Iancu-Agou, D. 1976. Préoccupations intellectuelles des médecins juifs au Moyen Âge : inventaires de bibliothèques. Provence historique t. 26, fasc. 103 : 21-44.

Kessler-Mesguich, S. 2013. Les études hébraïques en France : de François Tissard à Richard Simon, (1508-1680). Travaux d'humanisme et Renaissance 517. Genève : Librairie Droz.

Kogel, J. 2018. Le Ma'aseh efod ou l'apprentissage de la langue hébraïque en solitaire. Iberia judaica, $\mathrm{n}^{\circ} 10: 21-32$.

Kozodoy, M. 2015. The secret faith of Maestre Honoratus. Profayt Duran and Jewish identity in Late Medieval Iberia. The Middle Ages Series. Philadelphie PA : University of Pennsylvania Press.

Renan, E. 1893. Les écrivains juifs français du XIVee siècle. Paris : Imprimerie nationale.

Richler, B. et Beit-Arié, M., éd. 2001. Hebrew manuscripts in the Biblioteca Palatina in Parma : Catalogue. Jérusalem : Hebrew University of Jerusalem, Jewish National and University Library.

Sáenz-Badillos. 2001. Hebrew philology in Sefarad : The state of the question. Dans Hebrew scholarship and the medieval world, éd. par N. de Lange, 38-59. Cambridge : Cambridge University Press.

Schwarzfuchs, L. 2008. L’hébreu dans le livre lyonnais au XVI siècle : inventaire chronologique. Métamorphoses du livre. Lyon : ENS Éditions/Institut d'histoire du livre.

Sela, Sh. et Freudenthal, G. 2006. Abraham ibn Ezra's scholarly writings : A chronological listing. Aleph, $\mathrm{n}^{\circ} 6: 13-55$.

\section{NOTES}

1. Friedländer (1865). Ce sujet a déjà été abordé dans une perspective différente, voir Kogel (2018). Pour des raisons de lisibilité, nous avons adopté une translitération simplifiée pour les noms propres, les titres d'œuvres, les noms de lettres, ainsi que les voyelles. En revanche, il nous a semblé nécessaire de distinguer entre les lettres $b, k$ et $p$ et leur réalisation fricative $\underline{b}, \underline{k}$ et $\bar{p}$ (et non $v$, kh et $f$ ) dans la traduction des différents extraits grammaticaux, pour permettre au lecteur de suivre plus aisément les explications de Profiat Duran et de Sante Pagnini.

2. Ces deux traités ont été traduits une première fois, au XI ${ }^{e}$ siècle, par Moïse Gikatilla, sous le titre Sefer otyot ha-nah we-ha-meshekh et Sefer ba'aley ha-kefel (Hayyuj 1870) et une seconde fois, à Rome vers 1140-1142, par Abraham ibn Ezra, sous le titre Sefer otyot ha-nah et Sefer pe'aley ha-kefel (Hayyuj 1844). En ce qui concerne la traduction d'Abraham ibn Ezra, voir l'article de Shlomo Sela et Gad Freudenthal (2006). 
3. On peut citer, par exemple, l'adaptation à l'hébreu du paradigme fa'ala. La première occurrence apparait dans les Těšubot des disciples de Menaḥem ben Saruq à Dunaš ben Labraṭ (Benavente Robles et Sáenz-Badillos 1986). L'utilisation des radicales $f \bar{a}$ ', 'ayn et lām pour désigner les trois lettres radicales est explicite dans l'ouvrage de Hayyuj. Pour l'adaptation en hébreu, c'est Ibn Gikatilla qui fait figure de pionnier (Hayyuj $1870: 5$ ).

4. Sáenz-Badillos (2001 : 47) ; la terminologie utilisée par Ḥayyuj est aussi largement empruntée à celle de la grammaire arabe (Bacher 1882).

5. Sáenz-Badillos (1986). Cet ouvrage a été précédé par les listes massorétiques, comme Sefer okhlah we-okhlah, qui regroupaient les mots selon des critères sémantiques ou morphologiques (Díaz Esteban 1975).

6. Point diacritique placé dans la consonne, dont on distingue deux types : le dageš léger qui marque la prononciation occlusive de certaines consonnes et le dageš fort qui note un redoublement. C'est de ce dernier qu'il s'agit ici.

7. Ḥayyuj (1870: 100). La traduction d'Abraham ibn Ezra est plus littérale et plus concise - il est possible qu'il ait disposé d'une version arabe différente - et en voici la dernière phrase (Hayyuj $1844: 146)$ : «si [la forme] est marquée d'un dageš, tu sauras avec certitude qu'il s'agit d'un verbe géminé, et sinon qu'il s'agit d'un verbe à deuxième radicale faible. »

8. «Le livre de la langue élégante » a été composé en tishre 4906 [octobre 1145] à Rome (Sela et Freudenthal $2006: 19)$.

9. Le Kitāb al-Tanqīh, traduit par by Judah ibn Tibbon, comprenait deux parties : une grammaire Kitāb al-Luma' et un dictionnaire des racines Kitāb al-uṣul, qui avaient pour titre, en hébreu, Sefer ha-Riqmah et Sefer ha-Shorashim (Ibn Janah 1896 et 1964).

10. J. Qimhi (1888), M. Qimḥi (1519) et D. Qimḥi (1966).

11. Iancu-Agou (1976).

12. C'est ce dont témoigne une des gloses relevées par Benjamin Richler dans le manuscrit Parma, Biblioteca palatina 2290: "c'est ce qu'il nous a transmis en secret et en se cachant $\left(f^{\circ} 23 v^{\circ}\right) »$. Voir Richler et Beit-Arié (2001 : notice 1310) et également le livre de Maud Kozodoy (2015).

13. Une lettre adressée en 1393 à Joseph ben Abraham à la mort de son père, R. Abraham ben Isaac ha-Levi de Gérone, un des guides spirituels les plus importants de la communauté juive catalane, dans laquelle il décrit la situation désespérée du judaïsme contemporain ; deux textes de polémique anti-chrétienne, une lettre satirique - 'Al tehi ke-avotekha, « Ne sois pas comme tes pères »- adressée en 1396 à son ami David Bonet Bonjorn, converti de force mais qui renia ensuite la foi de ses pères, et Kelimat ha-goyim, «L'opprobre des nations », généralement daté de 1397, dans lequel Profiat Duran s'attaque aux fondements du christianisme (Renan 1893 : 395-404). Selon Richard W. Emery (1968), ces ouvrages composés en hébreu étaient destinés à des juifs ou à des conversos, dans le but de les soutenir et de les encourager à persévérer dans leur foi. 14. Comme souvent, les titres des ouvrages hébreux rappellent des expressions bibliques, tout en fournissant des indications concernant l'auteur. Si dans la Bible, ep̄od (alef - pe - dalet) désigne un vêtement du grand-prêtre (Ex 28,15 et 39,8), il faut le lire ici comme l'acronyme de Amar Profiat Duran, « Profiat Duran a dit».

15. Un des sept schèmes verbaux ou binyanim 'constructions', comme les ont nommés les grammairiens médiévaux ; les noms des binyanim correspondent au croisement de la racine-type $\mathrm{P}^{\prime} \mathrm{L}$ avec le schème de l'accompli, troisième personne du masculin. On a ainsi le פa pa al ou qal 'le léger', qui ne comprend ni redoublement, ni ajout de consonne, le nip̄'al, le pi el, le pu'al, le hip̄'il, le hō̄'al, le hitpa'el.

16. Hayyuj (1870: 100, l. 23 et suivantes), cité ici p. 4.

17. Voir plus loin l'explication plus complète de Pagnini.

18. Pagnini (1526, Libri IIII, chap. 4 et 5, p. 395-400). Le titre de la première édition étant très long parce qu'il détaillait ce que contenaient les quatre livres de ces Hebraicas institutiones, les éditions 
ultérieures retiendront la mention figurant à la fin du livre, expliciunt Quatuor Libri Institutionum Hebraicarum (1549).

19. Les deux grammaires de l'hébreu imprimées précédemment en France étaient assez rudimentaires : Tissard (1508 ou 1509) ; Qimhi ([1520]).

20. Schwarzfuchs (2008: 16-19).

21. Voir Kessler-Mesguich (2013: 127 et n. 26).

22. La première édition du Mikhlol date de 1545 (Venise : Daniel Bomberg), tandis que le Ma'aseh efod n'a pas été publié avant 1865 .

23. Kessler-Mesguich (2013: 145) : «Le Ma'aseh efod n'est explicitement cité que cinq fois, à propos des différences de prononciation entre les différentes communautés juives, et à propos des conjugaisons passives ».

24. Ibid. : 138.

25. Dans l'édition de 1549, les chapitres 4 et 5 sont réunis sous le titre Modus inveniendi radices, hoc est themata, et primitiva omnium Hebraicarum dictionum avec comme sous-titre Ratio inveniendi radices nominum, « méthode pour trouver les racines des noms » (p. 434-437) pour le chapitre 4 et Ratio inveniendi radices verborum, "méthode pour trouver les racines des verbes» (p. 437-439) pour le chapitre 5 .

26. Thème latin = racine et le suffixe, sans les désinences personnelles. Pour un nom, on pourrait parler en hébreu de schèmes nominaux. Dans la préface du Dictionarium hebraicum, Münster désigne le "Livre des racines" dont il s'inspire, Sefer ha-shorashim de David Qimhi, par l'expression liber de Radicibus et il ajoute sic enim illa gens themata dictionum vocat « car c'est ainsi que ces gens appellent les thèmes des mots » $(1523, \mathrm{f}$. Aa2v).

27. Pagnini emploie plusieurs termes pour désigner les voyelles : nequdot, tenu'ot, rešamim et melakim (Hebraicarum institutionum : 6). Kessler-Mesguich (2013 : 132) fait remarquer que la présentation de Pagnini rassemble des termes métalinguistiques provenant de plusieurs grammairiens. Le terme melakim 'rois', bien qu'employé dans le Mikhlol de David Qimhi, renvoie au système le plus ancien, celui des sept rois, c'est à dire des sept timbres, auxquels s'oppose le « serviteur », le šewa (ibid., n. 132).

28. C'est un passage du $E f o d$ (Friedländer $1865: 141$ ).

29. Ces lettres permettent de former la phrase mnémotechnique que David Qimhi (1548, f. 38r) cite au nom de son frère Moïse : moše katab 'elenu « Moïse nous a écrit » en revanche, ce dernier a repris dans le Mahalakh celle qui figurait déjà dans le Maḥberet : etkenah mešali bo (Qimḥi, M. 1519 $: 6)$.

30. Ces lettres forment le mot he'emanti 'j'ai eu confiance'.

31. L'édition 1529 a un shin et non un אשבע tsade, une erreur corrigée dans celle de 1549.

32..dans l'édition de 1529 צוד

33. L'édition de 1529 comporte une phrase qui a été supprimée de l'édition de 1549 : «t dans Et 'ir 'ville', une fois enlevé le iod en puissance [et ער] signe de voyelle royale, il reste [1] chiric [est] $v a u$, et la racine est עוֹ 'WR."

34. Jr 30 : 13. La traduction de ces deux termes ne figure pas dans l'édition de 1529.

35. L'édition de 1529 a " מחילה mehillah 'commencement', une fois enlevées les lettres memet [מ] he.une erreur corrigée dans l'édition de 1549 , «[ [ה]

36. Pagnini (1529).

37. Friedländer (1865 : 144) : והדרך הששי.

38. Référence erronée dans Pagnini (1529).

39. Ibid. פִִִִּּ : pattīm.

40. La formulation est laconique. Les monosyllabiques ne dérivent pas de la forme plurielle mais bien de la racine géminée que l'on identifie grâce à la présence du dageš dans les dissyllabiques.

41. Buxtorf (1629 : f. 6r-v de l'introduction). Seuls quelques exemples supplémentaires des points 2 et 5 n'ont pas été reproduits ici. 
42. Buxtorf (1691 : 30-31) : «1. Enlève les serviles, et s'il reste trois lettres, tu peux considérer qu'elles forment la racine du thème. 2. Si deux lettres seulement restent, tu peux ajouter soit nun, soit yod au début, ou insérer un waw au milieu, ou encore mettre un he à la fin, ou doubler la deuxième lettre, ce qui permettra de trouver la racine recherchée. 3. S'il reste une lettre seulement, après avoir enlevé les serviles, tu dois mettre soit un nun soit un yod au début, et un he à la fin; la racine sera ainsi identifiée. 4 . Tout ce qui s'écarte de ces règles, l'expérience, cher lecteur, te le fournira.»

43. James Noble (1832 : 122-124) cite le compendium de Adrianus Reeland (1721) et bien sûr l'ouvrage de Johannes Buxtorf (1629 : f. 6r-v de l'introduction), maintes fois édité. La filiation entre ces ouvrages est indiquée par les auteurs. Voir également Sámuel Zsigmondy (1828:90) où ne figurent que les trois premières règles précédées de la mention Antiquiores Grammatici secundum hos versus verborum radicem investigabant.

44. Ladvocat (1755).

\section{RÉSUMÉS}

La notion de trilitéralité des racines, fortement inspirée par la tradition arabe, a demandé une grande créativité pour être mise en œuvre dans la grammaire hébraïque. Judah Ḥayyuj (Fez, 950 - Cordoue, ca 1000) fit œuvre de pionnier en analysant le comportement des consonnes faibles qui peuvent ne pas être visibles dans certaines formes verbales tout en restant présentes dans la forme théorique de base. Ses travaux ont été poursuivis par Jonah ibn Janah (Cordoue, ca 985/990 - ca 1050) dont les ouvrages, adaptés ou traduits en hébreu, ont permis la diffusion des doctrines grammaticales de l'hébreu en Europe chrétienne et l'adoption définitive de la théorie des racines trilitères. Les dictionnaires des racines sur le modèle du Kitāb al-ușūl d'Ibn Janaḥ, outil commode pour classer le lexique biblique, devinrent populaires en Provence médiévale. Il restait cependant une difficulté majeure, à savoir les manières d'identifier la racine d'une forme nominale ou verbale complexe. Profiat Duran (Perpignan, < 1360 - ca 1414) fut le premier auteur à insérer dans sa grammaire, le $M a$ 'aseh efod, un chapitre décrivant les différentes méthodes permettant l'identification des racines. Ce passage, adapté ou résumé, fut fréquemment repris par les humanistes chrétiens dans leurs ouvrages linguistiques, et ce jusqu'au XIX ${ }^{\mathrm{e}}$ siècle.

The notion of triconsonantal roots was borrowed from the Arabic tradition and a great deal of creativity was required in order to apply it to Hebrew grammar. Judah Hayyuj (Fez, 950 Cordoba, $\mathrm{ca}$ 1000) was the first to note that weak consonants have a different comportment, that they may not be visible in certain verbal forms, but remain present in the theoretical basic form. His work was carried on by Jonah ibn Janah (Cordoba, ca 985/990 - ca 1050), whose writings were adapted or translated into Hebrew. This led to the diffusion of Hebrew grammatical knowledge in Christian Europe and the adoption of the theory of triconsonantal roots. Dictionaries of roots patterned on Ibn Janah's Kitāb al-ușūl, which are a convenient tool for classifying the lexicon of biblical words, became popular in medieval Provence. A major difficulty remained, namely how to identify the root of a complex nominal or verbal form. Profiat Duran (Perpignan $<1360-c a$ 1414) was the first author to include in his grammar, Ma'aseh efod, a chapter describing the different methods for identifying the roots. This chapter, through adaptations or summaries, was often used by the Christian humanists in their linguistic works down to the nineteenth century. 
INDEX

Mots-clés : Qimhị (David), identification de la racine en hébreu, Duran (Profiat), racines trilitères en hébreu, Pagnini (Sancte), tradition grammaticale hébraïque

Keywords : Qimhi (David), Hebrew grammatical tradition, identifying roots in Hebrew, Duran (Profiat), Pagnini (Sancte), triconsonantal roots in Hebrew

\section{AUTEUR}

JUDITH KOGEL

CNRS, Institut de recherche et d'histoire des textes (UPR 841 IRHT), Aubervilliers, France 